\title{
A prospective analysis of the cost-effectiveness of alfuzosin, tamsulosin and silodosin for 12 weeks in benign prostatic hyperplasia
}

\author{
Manjunatha R. ${ }^{1}$, Pundarikaksha H. P. ${ }^{1}$, Madhusudhana H. R. ${ }^{2}$
}

\author{
${ }^{1}$ Department of Pharmacology, \\ Kempegowda Institute of \\ Medical Sciences, Bangalore, \\ Karnataka, India \\ ${ }^{2}$ Department of Urology, \\ Kempegowda Institute of \\ Medical Sciences, Bangalore, \\ Karnataka, India
}

Received: 24 August 2016

Accepted: 24 September 2016

*Correspondence to:

Dr. Manjunatha R.,

Email:

manjunatha5ramaiah@gmail.co

$\mathrm{m}$

Copyright: () the author(s), publisher and licensee Medip Academy. This is an openaccess article distributed under the terms of the Creative Commons Attribution NonCommercial License, which permits unrestricted noncommercial use, distribution, and reproduction in any medium, provided the original work is properly cited.

\begin{abstract}
Background: Benign prostatic hyperplasia (BPH) is usually seen in men above 45 years. $\alpha$-blockers (alfuzosin, tamsulosin and silodosin) form the mainstay of pharmacological management of symptomatic BPH and may differ in their efficacy, tolerability and treatment costs. The present study compares them prospectively to evaluate the most cost-effective $\alpha$-blocker in the management of BPH.
\end{abstract}

Methods: Ninety subjects diagnosed with symptomatic BPH were randomised to receive alfuzosin, tamsulosin or silodosin and were followed up at 2, 4, 8 and 12 weeks after treatment initiation. Effectiveness was assessed by rate of treatment success and number of symptom free days (SFDs). Treatment related direct medical, direct non-medical and indirect costs were analysed both from patient and third-party perspective. Cost-effectiveness was assessed using average cost-effectiveness ratio (ACER) and incremental cost-effectiveness ratio (ICER).

Results: With rate of treatment success as the outcome measure, alfuzosin had the least ACER, followed by tamsulosin and silodosin. With number of SFDs as the outcome measure, alfuzosin had the least ACER followed by silodosin and tamsulosin. An additional INR 3982 and INR 30 were required per extra success and extra SFD respectively with alfuzosin when compared to tamsulosin. Alfuzosin dominated silodosin as a more cost-effective option in achieving treatment success. However, an additional INR 231 was required to achieve an extra SFD with silodosin.

Conclusions: Compared with tamsulosin and silodosin, alfuzosin seems to be the most economical $\alpha$-blocker in the management of BPH, both from patient and third-party perspective. Short duration of study of 12 weeks was a limitation in the present prospective study.

Keywords: ACER, Alfuzosin, Benign prostatic hyperplasia, Cost-effectiveness, ICER, Silodosin, Tamsulosin

\section{INTRODUCTION}

Lower urinary tract symptoms (LUTS) like increased frequency of micturition, urgency, nocturia, weak stream, intermittency, straining and incomplete emptying of the bladder are the symptoms commonly seen in patients with BPH. Treatment becomes necessary when the above symptoms interfere with day-to-day activities of an individual and also to avoid complications of the disease like hematuria, urinary tract infections (UTIs), acute urinary retention and kidney failure. , $^{1,2}$
The line of management of BPH, either medical or surgical, depends upon the patient profile and stage of the disease. $\alpha_{1}$ - receptor blocking drugs and $5 \alpha$-reductase inhibitors (5 $\alpha \mathrm{RI})$ forms the mainstay of medical management of $\mathrm{BPH}$. As $\alpha$ receptors have a varied distribution in body $\left(\alpha_{1 \mathrm{~A}}\right.$ : smooth muscle in the bladder neck and prostate; $\alpha_{1 \mathrm{~B}}$ : vascular smooth muscle, $\alpha_{1 \mathrm{D}}$ : bladder muscle), any drug with more selective action towards $\alpha_{1 \mathrm{~A}}$ receptors will be highly effective with minimum vascular side effects when compared to less selective $\alpha$ antagonists. ${ }^{1}$ 
Currently, three $\alpha$ blockers are commonly used in the management of BPH namely alfuzosin, tamsulosin and silodosin. Alfuzosin is a non-selective $\alpha$ blocker. However, studies have shown that it has minimum effects on hemodynamics. ${ }^{2}$ Tamsulosin has a lower risk of vascular side effects as it is selective for $\alpha_{1 \mathrm{~A}}$ and $\alpha_{1 \mathrm{D}^{-}}$ receptors. The affinity of tamsulosin for $\alpha_{1 \mathrm{~A}}$ receptors is 10 times greater than that for $\alpha_{1 \mathrm{~B}}$ receptors. ${ }^{1}$ Silodosin is the latest addition among ablockers. The affinity of silodosinfor $\alpha_{1 \mathrm{~A}}$ receptors is 162 times greater than those for $\alpha_{1 \mathrm{~B}}$ receptors and 50 times greater than that for $\alpha_{1 \mathrm{D}}$ receptors.Its action on afferent nerves of the urinary bladder has been hypothesized to control the overactive symptoms like frequency, urgency and nocturia. ${ }^{1}$

Pharmacoeconomic analyses between medical therapies of different mechanisms of action and between medical and surgical therapies have been conducted. ${ }^{3-9}$ However, data on pharmacoeconomic analysis comparing the present-day commonly used newer $\alpha_{1}$ blockers is lacking, which may differ in selectivity of action, effectiveness rate, safety profile, and associated cost. Consideration of cost-effectiveness analysis (CEA) can help to quantify potential advantages of alfuzosin, tamsulosin and silodosin and facilitate treatment choices.

\section{METHODS}

This study was designed as a parallel group study comparing three $\alpha$ blockers in an open label fashion by randomising 90 subjects attending the Urology outpatient department (OPD), Kempegowda Institute of Medical Sciences Hospital and Research Centre, Bengaluru who met the diagnosis of symptomatic BPH. The study was conducted between September 2013 and June 2014 and was registered with the CTRI bearing number CTRI/2013/10/004112.

Men with BPH and LUTS aged at least 45 years and have an International prostate symptom score (IPSS) of 8 or more, Quality of life score (QLS) 3 or more, peak flow rate $(\mathrm{Qmax})<15 \mathrm{ml} / \mathrm{s}$ but $>4 \mathrm{ml} / \mathrm{s}$ with a voided volume of $>100 \mathrm{ml}$ were included in the present study.

Relevant clinical and laboratory investigations were conducted to confirm the diagnosis of BPH as well as rule out complications of the disease and contraindications to study drugs. IPSS was used to assess the severity of LUTS as described elsewhere. ${ }^{10}$

IPSS was assessed at baseline and at the follow up visits after 2, 4, 8 and 12 weeks of treatment initiation by the investigator. The patients were instructed to carefully observe the severity of their urinary complaints and report the same when asked about it at the subsequent visit during the recording of the IPSS.

Out of the 90 randomized subjects, 30 received alfuzosin slow release (SR) 10 mgonce daily [Tab. Flotral $10 \mathrm{mg}$; Ranbaxy], 30 received tamsulosin 0.4 mgonce daily [Tab.
Contiflo-Icon $0.4 \mathrm{mg}$; Ranbaxy.] and 30 received silodosin 8 mgonce daily [Tab. Silofast $8 \mathrm{mg}$; Cipla].

Costs consideredin performing the cost-effectiveness analysis. $^{13,14}$

\section{Direct medical costs}

OPD card, pre and post void ultrasonography of kidney, ureters and bladder (USG KUB), serum PSA, serum creatinine, electrocardiography (ECG), uroflowmetry, urine routine, ascending urethrogram (ASU), micturating cystourethrogram (MCU), drug acquisition, unscheduled visits (inclusive of hospital charges, transportation charges, loss of wages of patient and caretaker), treatment of adverse events (drugs, supplies, hospital bills).

\section{Direct non-medical cost}

Cost of transportation to the hospital. Though the bus fare is charged stage wise and not kilometre wise, the roundtrip travel cost was calculated considering the average bus fare as:Bangalore metropolitan transport corporation (BMTC) charges of INR $2 / \mathrm{km}$ for patients from Bangalore and Karnataka state road transport corporation (KSRTC) charges INR $1.5 / \mathrm{km}$ for patients from outside Bangalore, for both patient and caretaker. This was kept constant for all the subjects recruited in the present study. $^{14}$

\section{Indirect cost}

Indirect cost included loss of wages to the patient and caretaker. Loss of wages of patients per day was calculated as per their monthly income. The pension of those patients who had retired from service was not included in the analysis. As per minimum wages and variable dearness allowance given by Ministry of Labour, Government of Karnataka, applicable to the time period on which the subjects were recruited for the study, the loss of wages of the caretaker were calculated. ${ }^{15}$

The medications used in each treatment group were of the same brand and cost per unit of study drug was taken from thestandard hospital pharmacy retail price list. The cost-effectiveness was analyzed using ACER both from patient and third party (Hospital / Insurance Company) perspective and the values were plotted on the costeffectiveness plane. ICER was calculated for those alternatives whose co-ordinates fell in quadrant I or III of the cost-effectiveness plane. ${ }^{13,14}$ Medical and non-medical costs were measured in terms of Indian National Rupee and clinical outcome in terms of treatment success (number of patients with $\geq 25 \%$ improvement in IPSS from baseline ${ }^{[16],[17]}$ ) and SFDs (number of days with IPSS $\leq 7$ ) during the three month treatment period. A patient maintaining IPSS at $\leq 7$ with the on-going treatment is considered to be adequately responding without warranting requirement of any change in therapy. Number of days with IPSS $\leq 7$ during the study period 
was considered as SFDs. Earlier the above mark achieved, greater will be the number of SFDs. SFDs as an outcome measure reflects the rapidity of onset of drug action. While the rate of treatment success as an outcome measure reflects the number of patients obtaining and maintaining satisfactory improvement during the 3 month treatment period. Thus both have their own role as outcome measures and assessing and comparing them separately in the present study reflects the two different aspects of benefits obtained by the patients for the money being spent on treatment. All the adverse events were recorded and assessed for causality as per the World Health Organisation- uppsala monitoring centre (WHOUMC) criteria and the cost of treatment of adverse events (AEs) (inclusive of the cost of additional investigation, drugs, travel and loss of wages of patient and caretaker due to AEs) with causality as either certain, probable or possible were included in the analysis. ${ }^{18}$

Following institutional ethics committee approval the study was started in September 2013, and written informed consent was obtained from all participants.

\section{Statistical methods}

\section{Sample size calculation}

There are no studies conducted yet in India comparing the effectiveness of alfuzosin, tamsulosin and silodosin in the management of BPH. From the latest previous published literature, tamsulosin has a treatment success rate of $82 \%$ and silodosin, $86 \% .{ }^{17}$ As there are no studies demonstrating the treatment success rate of alfuzosin with $\geq 25 \%$ improvement in IPSS as the criteria for treatment success, we conducted a pilot study with 12 patients. All the patients met the criteria of treatment success and thus the rate of success was taken as $99 \%$ for the purpose of calculation of sample size. With the noninferiority criteria of 5\%, 2-sided alpha at 5\% and chances of type-II error at $15 \%$ (Power of $85 \%$ ) and dropout rate at 5\%, 25 subjectswere required in each groupfor cost-effectiveness comparison between alfuzosin and tamsulosin groups and 30 subjects in each group for costeffectiveness comparison between alfuzosin and silodosin groups. Thus, a uniform number of 30 subjects in each group (alfuzosin, tamsulosin and silodosin) were recruitedfor the present study.

\section{Tests}

The CEA was done by ACER and ICER using the formulae: ${ }^{14}$

\section{$\mathrm{ACER}=\quad$ Health care costs (INR) \\ Clinical outcome (probability of treatment success or number of SFDs)}

ICER $=\quad$ Cost of drug A- Cost of drug B Success rate or SFDs with drug A- Success rate or SFDs with drug $B$

\section{RESULTS}

Of the 115 patients screened, 90 met the selection criteria and were randomly assigned to three treatment groups in $1: 1: 1$ ratio to receive alfuzosin, tamsulosin or silodosin. None withdrew from the study and there were no protocol violations. All the 30 patients in each group completed the study and were included for analysis. The demographic and baseline characteristics of the subjects wascomparable across the three treatment groups. Patients in the alfuzosin, tamsulosin and silodosin groups had a mean age of $63.43 \pm 8.91,63.60 \pm 9.05$ and $64.00 \pm 11.14$ years and a baseline IPSS of 19.2 \pm 9.6 , $21.63 \pm 7.63$ and $15.93 \pm 6.03$ respectively. Table 1 shows the unit and total direct medical costs (drug acquisition, consultation, investigations and treatment of AEs) incurred during the three month treatment period.

Patients in the alfuzosin, tamsulosin and silodosin groups had a treatment success rate of $100 \%, 93.3 \%$ and $96.7 \%$, and SFDs of approximately 56, 46 and 57 per patient respectively. The cost of treatment per patient was approximately INR 4974, 4696 and 5513 from the patient's perspective and INR 3696, 3635 and 4420 from the third partyperspective in the alfuzosin, tamsulosin and silodosin groups respectively (Table 2). With rate of treatment success as the clinical outcome, alfuzosin had the least ACER (INR 4974 from patient perspective and 3696 from third party perspective), followed by tamsulosin (INR 5033 from patient perspective and 3896 from third party perspective) and silodosin (INR 5701 from patient perspective and 4571 from third party perspective). With number of SFDs as the clinical outcome, alfuzosin had the least ACER (INR 90 from patient perspective and 67 from third party perspective) followed by silodosin (INR 96 from patient perspective and 77 from third party perspective) and tamsulosin (INR 103 from patient perspective and 79.5 from third party perspective) (Table 3). Cost effectiveness planes (Figure 1 , panels- A, B, C and D) were constructed to observe the relationship between the differences in the cost and clinical outcomes between the treatment groups and the necessity to conduct ICER. ${ }^{13}$ Alfuzosin showed a better outcome (both in terms of effectiveness and SFDs) and required higher spending than tamsulosin, with the coordinates falling in the quadrant $I$ of the costeffectiveness plane. Though the cost per patient is less for tamsulosin (INR 4695.66) than alfuzosin (INR 4974.41), alfuzosin has better ACER due to its higher treatment success rate and higher number of SFDs. Thus, ICER was calculated to know the additional cost that has to be spent on the most cost-effective treatment option (alfuzosin in this case) to increase success rate by $1 \%$ and increase SFD by 1 day, over and above that required for tamsulosin. An additional INR 3982 was required per extra success and an additional INR 30 was required per extra SFD with alfuzosin when compared to tamsulosin. On comparison of alfuzosin w.r.t silodosin, the coordinate for the rate of treatment success against cost fell in the quadrant II, i.e., higher effectiveness with lower 
cost, indicating that alfuzosin dominates silodosin as a more cost-effective option in achieving treatment success. Whereas, the co-ordinate for SFDs against cost for the comparison between the above two groups fell in the quadrant III, indicating both lesser SFDs and lesser cost with alfuzosin. Consequently, it was calculated that an additional INR 231 is required achieve an extra SFD with silodosin (Table 4).
The total number of AEs with causality assessment certain, probable and possible were 5,121 and 242 respectively. There were neither any serious adverse events nor treatment discontinuations. The most common AE was upper respiratory tract infectionseen in 14 subjects with alfuzosin, 10 with tamsulosin and 14 with silodosin.

Table 1: Direct medical costs in alfuzosin, tamsulosin and silodosin groups.

\begin{tabular}{|c|c|c|c|c|}
\hline \multirow{2}{*}{ Direct medical costs } & \multirow{2}{*}{ Unit cost (INR) } & \multicolumn{3}{|l|}{ Total cost (INR) } \\
\hline & & Alfuzosin $(\mathbf{n}=\mathbf{3 0})$ & Tamsulosin $(n=30)$ & Silodosin $(n=30)$ \\
\hline Tab Alfuzosin 10 mg* & 9.75 & 26325 & - & - \\
\hline Tab Tamsulosin 0.4 mg* & 9.72 & - & 26244 & - \\
\hline Cap Silodosin 8 mg* & 19 & - & - & 51300 \\
\hline OPD card $\dagger$ & 75 & 11400 & 11325 & 11325 \\
\hline USG KUB ${ }^{\ddagger}$ & 300 & 9000 & 9000 & 9000 \\
\hline Serum PSA ${ }^{\ddagger}$ & 150 & 4500 & 4500 & 4500 \\
\hline Serum creatinine ${ }^{\ddagger}$ & 60 & 1800 & 1800 & 1800 \\
\hline Electrocardiography & 75 & 2400 & 2250 & 2250 \\
\hline Uroflowmetry $^{\S}$ & 300 & 45000 & 45000 & 45000 \\
\hline Urine routine & 60 & 240 & 300 & 60 \\
\hline Cardiology OPD referral & 130 & 130 & - & - \\
\hline Check cystoscopy & 2000 & 2000 & 2000 & - \\
\hline $\begin{array}{l}\text { Fasting and post-prandial } \\
\text { blood sugar }\end{array}$ & 80 & 80 & - & - \\
\hline ASU & 969 & - & 969 & - \\
\hline $\mathrm{MCU}$ & 996 & - & 996 & - \\
\hline Urine culture and sensitivity & 75 & - & 75 & - \\
\hline Cost of treatment of AEs & - & 4697 & 1751 & 4675 \\
\hline \multicolumn{5}{|c|}{$\begin{array}{l}* \text { Total cost was calculated by multiplying unit cost by total no of days of use ( } 90) \text { for total no of patients (30) } \\
\dagger \text { Total cost was calculated by multiplying unit cost by no of visits for total no of patients ( } 30) \\
\text { † Total cost was calculated by multiplying unit cost by total no of patients (30) } \\
\S \text { Total cost was calculated by multiplying unit cost by no of visits (5) for total no of patients (30) }\end{array}$} \\
\hline \multicolumn{5}{|c|}{$\begin{array}{l}\text { PSA: Prostate Specific Antigen; USG: Ultrasonography; KUB: Kidney Ureter Bladder; OPD: Out- Patient Department; } \\
\text { ASU: Ascending Urethrogram; MCU: Maturating Cysto Urethrogram; AEs: Adverse events }\end{array}$} \\
\hline
\end{tabular}

Table 2: Efficacy and cost comparisons.

\begin{tabular}{|c|c|c|c|}
\hline Outcome & Alfuzosin $(n=30)$ & Tamsulosin $(\mathrm{n}=\mathbf{3 0})$ & Silodosin $(n=30)$ \\
\hline \multicolumn{4}{|l|}{ 3-month clinical outcome } \\
\hline No. $(\%)$ of patients with $\geq 25 \%$ improvement in IPSS & $30(100)$ & $28(93.3)$ & $29(96.7)$ \\
\hline Total number of SFDs & 1652 & 1372 & 1722 \\
\hline No. of SFDs / subject* & 55.07 & 45.73 & 57.40 \\
\hline$\%$ of SFDs / subject & 61.19 & 50.81 & 63.78 \\
\hline \multicolumn{4}{|c|}{ 3-month cost (INR) } \\
\hline \multicolumn{4}{|c|}{ Total cost (inclusive of all direct medical, direct non-medical and indirect costs) } \\
\hline Patient perspective & 149232.26 & 140869.68 & 165378.08 \\
\hline Third party perspective & 110871.79 & 109060.68 & 132610.08 \\
\hline \multicolumn{4}{|l|}{ Cost per patient } \\
\hline Patient perspective & 4974.41 & 4695.66 & 5512.6 \\
\hline Third party perspective & 3695.73 & 3635.36 & 4420.34 \\
\hline \multicolumn{4}{|c|}{$\begin{array}{l}\text { *The duration of follow up for each patient was } 12 \text { weeks ( } 84 \text { days). } 30 \text { subjects were included in each study group } \\
\text { giving a total of } 360(12 \times 30) \text { weeks / } 2520 \text { ( } 360 \text { X 7) days of follow up. From these } 2520 \text { days, number of days with } \\
\text { IPSS } \leq 7 \text { was noted as SFDs and the total number of SFDs was divided by } 30 \text { to calculate the number of SFDs per subject. }\end{array}$} \\
\hline \multicolumn{4}{|c|}{ IPSS: International Prostate Symptom Score; SFD: Symptom Free Days } \\
\hline
\end{tabular}


Table 3: Calculation of ACER.

\begin{tabular}{|llllll|}
\hline $\begin{array}{l}\text { Patient perspective } \\
\text { Treatment } \\
\text { group }\end{array}$ & $\begin{array}{l}\text { Treatment } \\
\text { success }\end{array}$ & $\begin{array}{l}\text { SFDs / } \\
\text { patient }\end{array}$ & $\begin{array}{l}\text { Cost / patient } \\
\text { (INR) }\end{array}$ & $\begin{array}{l}\text { ACER [Average cost (INR) / } \\
\text { success] }\end{array}$ & $\begin{array}{l}\text { ACER [Average cost } \\
\text { (INR) / SFD] }\end{array}$ \\
\hline Alfuzosin & $100 \%$ & 55.07 & 4974.41 & $4974.41 / 1=4974.41$ & 90.33 \\
\hline Tamsulosin & $93.3 \%$ & 45.73 & 4695.66 & $4695.66 / 0.933=5032.86$ & 102.68 \\
\hline Silodosin & $96.7 \%$ & 57.40 & 5512.60 & $5512.60 / 0.967=5700.72$ & 96.04 \\
\hline \multicolumn{2}{l}{ Third party perspective } & & & & 67.11 \\
\hline Alfuzosin & $100 \%$ & 55.07 & 3695.73 & 3695.73 & 79.50 \\
\hline Tamsulosin & $93.3 \%$ & 45.73 & 3635.36 & 3896.42 & 77.01 \\
\hline Silodosin & $96.7 \%$ & 57.40 & 4420.34 & 4571.19 & \\
\hline
\end{tabular}

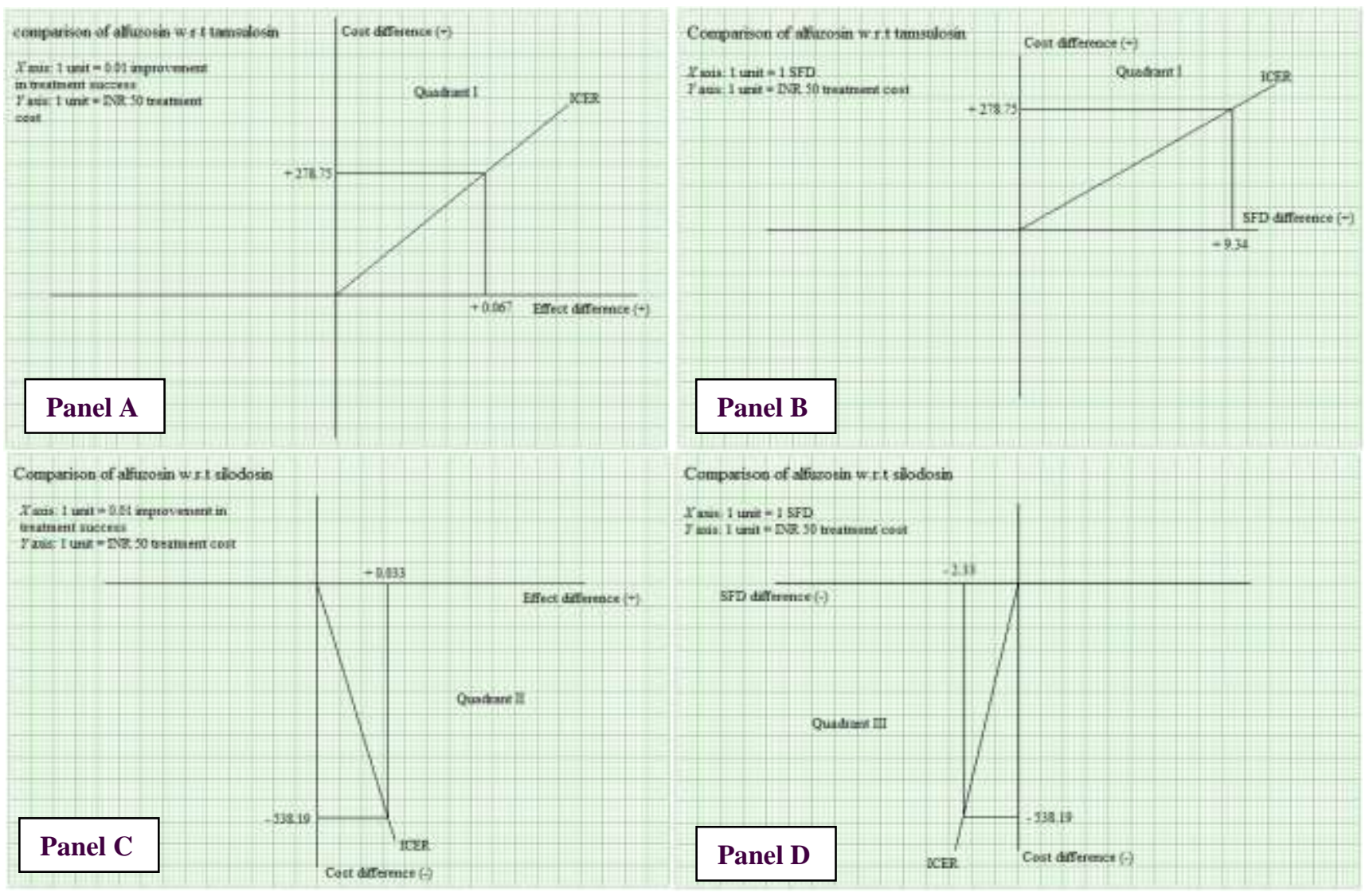

Figure 1: Cost-effectiveness planes showing the position of co-ordinates for various comparisons (patient perspective).

Figure 1-Panel A: Comparison of alfuzosin w.r.t tamsulosin taking rate of treatment success as the measure of effectiveness.

Figure 1-Panel B: Comparison of alfuzosin w.r.t tamsulosin taking SFDs as the measure of effectiveness.

Figure 1-Panel C: Comparison of alfuzosin w.r.t silodosin taking rate of treatment success as the measure of effectiveness.

Figure 1-Panel D: Comparison of alfuzosin w.r.t silodosin taking SFDs as the measure of effectiveness.

\section{DISCUSSION}

The present study shows that alfuzosin, with the least ACER per success and per SFD from patient and third party perspective, works out to be the most cost-effective $\alpha$ blocker in the treatment of BPH when compared to tamsulosin and silodosin. However, as alfuzosin yielded better results with higher spending per patient than tamsulosin, the ICER conducted showed an additional spending of $\sim$ INR 3982 / success and $\sim$ INR 30 / extra SFD from patient's perspective. 
Table 4: Calculation of ICER (patient perspective).

\begin{tabular}{|c|c|c|}
\hline $\begin{array}{l}\text { Treatment } \\
\text { groups }\end{array}$ & $\begin{array}{l}\text { ICER for } \\
\text { treatment success }\end{array}$ & ICER for SFDs \\
\hline $\begin{array}{l}\text { Alfuzosin } \\
\text { vs } \\
\text { tamsulosin }\end{array}$ & $\begin{array}{l}4974.41-4695.66 / \\
1-0.93=\text { INR } \\
3982.14 \text { per extra } \\
\text { success with } \\
\text { alfuzosin }\end{array}$ & $\begin{array}{l}4974.41-4695.66 / \\
55.07-45.73=I N R \\
29.84 \text { per extra SFD } \\
\text { with alfuzosin }\end{array}$ \\
\hline $\begin{array}{l}\text { Alfuzosin } \\
\text { vs } \\
\text { Silodosin }\end{array}$ & $\begin{array}{l}\text { Alfuzosin } \\
\text { dominates } \\
\text { silodosin }\end{array}$ & $\begin{array}{l}5512.60-4974.41 / \\
57.40-55.07= \\
230.98 \text { per extra } \\
\text { SFD with silodosin }\end{array}$ \\
\hline
\end{tabular}

Alfuzosin dominated silodosin in providing a better treatment success rate with lesser spending. Interestingly, silodosin seemed to provide a higher number of SFDs / patient ( $\sim 57$ days) than alfuzosin ( $\sim 55$ days). This discrepancy in efficacy may be due to earlier onset of action and lower baseline mean IPSS in silodosin group compared to alfuzosin group, as a SFD was defined as a day with IPSS $\leq 7 .^{1,2}$ Thus, silodosin with higher number of SFDs and higher spending per patient showed an additional spending of $\sim$ INR 231 per extra SFD when compared to alfuzosin. The better cost-effectiveness of alfuzosin is attributable to its higher efficacy and lower drug acquisition cost. Most of the previous pharmacoeconomic studies conducted earlier on medical therapy of $\mathrm{BPH}$ are retrospective in nature, with many using quality adjusted life years (QALY) as their outcome measure in their cost-utility analyses. ${ }^{3,4}$ In one of the studies conducted in the USA, with a time horizon of 20 years, alpha blockers and transurethral resection of prostate (TURP) were found to be the cost-effective options from the perspective of a US payer in the management of moderate to severe BPH with QALY as the outcome measure. However, transurethral microwave therapy was considered a dominant alternative in older patients with more severe disease. ${ }^{3}$ A study conducted in the UK comparing the cost-effectiveness of tamsulosin monotherapy with tamsulosin-dutasteride combination therapy with QALY as the outcome measure found that combination therapy had a high probability of being costeffective. ${ }^{4}$ Severalsuch studies have compared the costeffectiveness of monotherapy with $\alpha$ blockers versus their combination with $5 \alpha \mathrm{RI}$ and have shown that combination therapy is more cost-effective than monotherapy., Studies have also been conducted comparing the costeffectiveness of surgical modalities with medical management and have shown that minimally invasive surgeries and trans-urethral resection of prostate are either comparable or more cost-effective than medical therapy, with age and symptom severity as strong predictors of cost-effectiveness. ${ }^{3,7-9}$ A Swedish study comparing feedback microwave thermotherapy with alpha blockers for cost-effectiveness found that feedback microwave thermotherapy had a better cost-utility over a longer period of time when compared to alpha blocker therapy. ${ }^{7}$ A Canadian study comparing alpha blockers, 5 $\alpha \mathrm{RI}$ and TURP in BPH management found that the cost- effectiveness of alpha blockers was higher than that of 5 $\alpha R I$ and was comparable to that of TURP. ${ }^{8}$ A study conducted in the USA comparing watchful waiting, pharmacotherapy, surgery and combination of the above treatments found that surgery was a more cost-effective option in younger individuals while pharmacotherapy had better cost advantage in older individuals. ${ }^{9}$ This prospective randomised study had a few limitations. The duration of follow-up was short, for a period of 12 weeks only. The present study doesn't compare between tamsulosin and silodosin for their cost-effectiveness as the sample size was not adequately powered to do so. This was an open label, single centre study. Conduct of multicentric, blinded, long term studies with suitable modelling and sensitivity analysis, and sample size adequately powered to compare between tamsulosin and silodosin for their cost-effectiveness will add further to the existing data on the cost-effectiveness of $\alpha$ blocker therapy in the management of BPH. Cost-utility analyses, though cumbersome, may be conducted on similar lines to compare the $\alpha$ blockers, considering patient perceived improvements in QALY as a better and holistic measure of effectiveness.

\section{CONCLUSION}

All the three $\alpha$ blockers have shown to be effective in alleviating the LUTS associated with mild to moderate BPH. However, compared with tamsulosin and silodosin, alfuzosin seems to be the most cost-effective $\alpha$ blocker in the management of BPH, both from patient and third party perspective. Short duration of follow-up of 12 weeks was a limitation in the present prospective study.

\section{ACKNOWLEDGEMENTS}

Dr Amarkumar J and Dr Hanumantharaju BK, Consultant Urologists, KIMS Hospital and Research Centre, V V Puram, Bangalore, facilitated the patient recruitment and guided the present study as subject experts. Dr Vikram K Reddy, Tutor, Department of Pharmacology, Kempegowda Institute of Medical Sciences, Bangalore shared his ideas in designing the study methodology.

Funding: No funding sources Conflict of interest: None declared

Ethical approval: The study was approved by the Institutional Ethics Committee

\section{REFERENCES}

1. Rossi M, Roumeguère T. Silodosin in the treatment of benign prostatic hyperplasia. Drug Des Devel Ther. 2010;4:291-7.

2. Yoshida M, Kudoh J, Homma Y, Kawabe K. Safety and efficacy of silodosin for the treatment of benign prostatic hyperplasia. ClinInterv Aging. 2011;6:16172.

3. Di Santostefano RL, Biddle AK, Lavelle JP. The long-term cost effectiveness of treatments for benign 
prostatic hyperplasia. Pharmacoeconomics. 2006;24(2):171-91.

4. Walker A, Doyle S, Posnett J, Hunjan M. Costeffectiveness of single-dose tamsulosin and dutasteride combination therapy compared with tamsulosin monotherapy in patients with benign prostatic hyperplasia in the UK. BJU Int. 2013;112(5):638-46.

5. Ismaila A, Walker A, Sayani A, Laroche B, Nickel JC, Posnett J, et al. Cost-effectiveness of dutasteridetamsulosin combination therapy for the treatment of symptomatic benign prostatic hyperplasia: A Canadian model based on the Comb AT trial. Can UrolAssoc J. 2013;7(5-6):E393-401.

6. Takayama T, Arakawa I, Kakihara H, Tachibana K, Ozono S. Pharmacoeconomic evaluation of combination therapy with dutasteride and $\alpha 1$ blocker for treatment of benign prostatic hyperplasia in Japan. Hinyokika Kiyo. 2012;58(2):61-9.

7. Tennvall RG, Hjelmgren J, Malmberg L. Under what conditions is feedback microwave thermotherapy (ProstaLund Feedback Treatment) cost-effective in comparison with alpha-blockade in the treatment of benign prostatic hyperplasia and lower urinary tract symptoms? Scand J UrolNephrol. 2006;40(6):495505.

8. Nickel JC. BPH: costs and treatment outcomes. Am J Manag Care. 2006;12(5):S141-8.

9. Chirikos TN, Sanford E. Cost consequences of surveillance, medical management or surgery for benign prostatic hyperplasia. $\mathrm{J}$ Urol. 1996;155(4):1311-6.

10. Manjunatha R, Pundarikaksha HP, Madhusudhana $H$ R, Amarkumar J, Hanumantharaju BK. A randomized, comparative, open-label study of efficacy and tolerability of alfuzosin, tamsulosin and silodosin in benign prostatic hyperplasia. Indian $\mathbf{J}$ Pharmacol. 2016;48:134-40.

11. Mc Nicholas TA, Kirby RS, Lepor H. Evaluation and nonsurgical management of benign prostatic hyperplasia. In: Wein AJ, kavoussi LR, Partin AW, Novick AC, Peters CA, editors. Campbell-Walsh urology. 10th edition. Philadelphia, PA: Saunders; 2012:2611-2654.

12. Tanguay S, Awde M, Brock G, Casey R, Kozak J, Lee $\mathrm{J}$, et al. Diagnosis and management of benign prostatic hyperplasia in primary care. Can Urol Assoc J. 2009;3(2):S92-S100.

13. Walley $\mathrm{T}$, Chapter 9. Pharmacoeconomics and Economic Evaluation of Drug Therapies. [Updated 2012 July 13]. Available at: http://www.iuphar.org/pdf/hum_67.pdf. Accessed on March 1, 2013

14. Reddy VK, Girish K, Lakshmi P, Vijendra R, Kumar A, Harsha R. Cost-effectiveness analysis of baclofen and chlordiazepoxide in uncomplicated alcoholwithdrawal syndrome. Indian $\mathrm{J}$ Pharmacol 2014;46:372-7.

15. Department of Labour Karanataka [homepage on the internet]. Bengaluru: Department of labour; Notifications on minimum wages; [updated 2014 $\begin{array}{llll}\text { Aug } & \text { Available from: }\end{array}$ http://labour.kar.nic.in/labour/notification.htm. Accessed on September 20, 2014.

16. Ohsfeldt RL, Kreder KJ, Klein RW, Chrischilles EA. Cost-effectiveness of tamsulosin, doxazosin, and terazosin in the treatment of benign prostatic hyperplasia. J Manag Care Pharm. 2004;10:412-22.

17. Yu HJ, Lin AT, Yang SS, Tsui KH, Wu HC, Cheng $\mathrm{CL}$, et al. Non-inferiority of silodosin to tamsulosin in treating patients with lower urinary tract symptoms (LUTS) associated with benign prostatic hyperplasia (BPH). BJU Int. 2011;108:1843-8.

18. The Uppsala monitoring centre [homepage on the internet]. Uppsala, Sweden: Pharmacovigilance; [updated 2013 Jan 30] Available from: http://whoumc.org/Graphics/26649.pdf. Accessed on March 1, 2013.

Cite this article as: Manjunatha $\mathrm{R}$, Pundarikaksha HP, Madhusudhana HR. A prospective analysis of the cost-effectiveness of alfuzosin, tamsulosin and silodosin for 12 weeks in benign prostatic hyperplasia. Int J Basic Clin Pharmacol 2016;5:24817. 